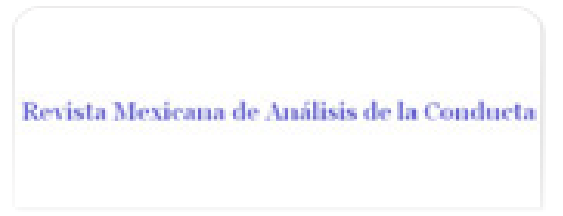

Revista Mexicana de Análisis de la Conducta ISSN: 0185-4534

editora@rmac-mx.org

Sociedad Mexicana de Análisis de la Conducta México

Martínez, Alma Gabriela; López-Espinoza, Antonio

Efectos post-privación con dos alternativas energéticas en ratas

Revista Mexicana de Análisis de la Conducta, vol. 33, núm. 1, junio, 2007, pp. 43-59

Sociedad Mexicana de Análisis de la Conducta

Guadalajara, México

Disponible en: http://www.redalyc.org/articulo.oa?id=59333104

Cómo citar el artículo

- Número completo

- Más información del artículo

- Página de la revista en redalyc.org

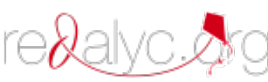

Sistema de Información Científica

Red de Revistas Científicas de América Latina, el Caribe, España y Portugal Proyecto académico sin fines de lucro, desarrollado bajo la iniciativa de acceso abierto 


\title{
EFECTOS POST-PRIVACIÓN CON DOS ALTERNATIVAS ENERGÉTICAS EN RATAS ${ }^{1}$
}

\author{
POST-DEPRIVATION EFFECTS WITH TWO ENGERGETIC \\ ALTERNATIVES IN RATS \\ ALMA GABRIELA MARTÍNEZ Y ANTONIO LÓPEZ-ESPINOZA² \\ UNIVERSIDAD DE GUADALAJARA
}

\begin{abstract}
RESUMEN
Veinticuatro ratas Wistar (3 meses de edad) se dividieron en cuatro grupos y fueron expuestas a libre acceso a alimento y agua durante quince días, seguidos de tres días de privación de alimento. Posteriormente cada grupo mantuvo libre acceso a alimento y a una de tres soluciones de agua con glucosa durante cinco días. La primera solución proporcionó $45 \mathrm{~g}$ de glucosa/200ml de agua (180 calorías), la segunda $30 \mathrm{~g}$ de glucosa/200ml (120 calorías) y la tercera $15 \mathrm{~g}$ de glucosa/200ml (60 calorías). El grupo control no recibió ninguna solución de agua con glucosa. El contenido energético del alimento no fue modificado. Los resultados mostraron que variar el contenido calórico del agua modifica la conducta alimentaria posterior a periodos de privación de alimento.

Palabras clave: calorías, glucosa, privación de alimento, periodo post-privación, peso corporal, consumo de alimento, consumo de agua, ratas.

1. Esta investigación fue financiada por la Beca 180463 y el Proyecto $46083-\mathrm{H}$ del CONACyT

2. Para correspondencia dirigirse a: Alma Gabriela Martínez, e-mail: almagabrielamm@yahoo.com mx. Antonio López-Espinoza, e-mail: anton779@megared.net.mx. Centro de Estudios e Investigaciones en Comportamiento. Universidad de Guadalajara. Francisco de Quevedo \# 180, Col. Arcos Vallarta, CP. 44500, Guadalajara, Jalisco, México
\end{abstract}




\begin{abstract}
Twenty-four albino rats (3-month-old at the beginning of the experiment) divided in four groups, were exposed to fifteen days of free access to water and food, followed by 3 days of food deprivation. Next five days every group was exposed to one of three kinds of water concentration of glucose. First concentration had $45 \mathrm{~g}$ of glucose $/ 200 \mathrm{ml}$ of water (180 calories), second $30 \mathrm{~g}$ of glucose $/ 200 \mathrm{ml}$ of water (120 calories), and third $15 \mathrm{~g}$ of glucose $/ 200 \mathrm{ml}$ of water (60 calories). Control group never received water concentration of glucose. Caloric food concentration remained the same. Food was available at all times during free access periods. Results suggest that modification of caloric water concentration affect the feeding behavior after food deprivation period.
\end{abstract}

Key words: calories, glucose, food deprivation, post-deprivation period, body weight, water and food consumption, rats.

\title{
EFECTOS POST-PRIVACIÓN CON DOS ALTERNATIVAS ENERGÉTICAS EN RATAS
}

Una característica común en el estudio de los trastornos alimentarios es la restricción de comida. El efecto que produce la aplicación de un periodo de restricción alimentaria sobre el peso corporal y los consumos de alimento y agua ha sido evaluado desde la perspectiva conductual por diversos investigadores (Baker, 1955; Lawrence y Mason, 1955; Polivy y Herman, 1985; Siegel y Stuckey, 1947). López-Espinoza (2001, 2004a, 2004b) y López-Espinoza y Martínez (2001a, 2001b, 2004) afirmaron que después de un periodo de restricción alimentaria, las ratas presentan cambios conductuales bien definidos a los que denominaron efectos post-privación. Reportaron que después de aplicar programas de privación de agua o alimento y de alternarlos con periodos de libre acceso, los sujetos experimentales recuperaron el peso corporal perdido durante la restricción y su consumo de comida y agua fue mucho mayor en los periodos post-privación respecto al consumo registrado durante la línea base.

López-Espinoza (2001, 2004a, 2004b) y López-Espinoza y Martínez (2001a, 2001b, 2004) manipularon diferentes variables para evaluar los cambios en la ocurrencia de los efectos post-privación. A pesar de modificar la duración del periodo de privación, el elemento privado o la edad de los sujetos, los efectos post-privación se presentaron sin cambios. López-Espinoza y Martínez (2004) señalaron que la teoría de regulación de Keesey (1986) basada en la existencia de un set-point es una posible explicación para sus resultados. 
La teoría del set-point (Keesey, 1986; Staddon y Zanutto, 1998) señala que después de un periodo de privación, los organismos compensarán la energía perdida consumiendo más calorías de las que consumían anteriormente, lo que explicaría las grandes comilonas y las grandes bebidas reportadas por López-Espinoza y Martínez (2001a, 2001b y 2004). Corwin, Wojnicki, Fischer, Dimitrou, Rice y Young (1998) reportaron que cuando los organismos se encuentran en un periodo de restricción de comida, también se encuentran en un periodo de restricción energética. Estos autores señalaron que existe una relación entre el contenido energético de los alimentos y la conducta de comer.

Collier, Hirsh y Kanareck (1983) demostraron experimentalmente que las ratas pueden regular su consumo de calorías. Ofrecieron a los sujetos alimentos con diversas cantidades de calorías y evaluaron la cantidad de alimento consumido y el total de calorías ingeridas. Señalaron que a pesar de la diversidad de alimentos disponibles, las ratas mantuvieron constante la cantidad de calorías que habitualmente consumen. Este fenómeno ha sido llamado regulación alimentaria (Capaldi, 1996; Collier, Hirsh y Kanareck, 1983; Lane, Ingram y Roth, 1999; Staddon y Zanutto, 1998; Strubbe y Woods, 2004; Weingarten, 1990). Por su parte, Anliker y Mayer (1957) mencionaron que las ratas de laboratorio entrenadas en presionar una tecla para obtener comida muestran regularidad en el consumo de calorías. Concluyeron que las ratas tienen ritmos de alimentación definidos de 24 horas. Mayer (1955), Keesey (1986), y Staddon y Zanutto (1998) señalaron que esta periodicidad del patrón alimentario de la rata es producto de la regulación de energía.

Collier, Hirsh y Kanareck (1983) sugirieron que ante cambios energéticos ambientales, las ratas modifican sus patrones alimentarios para regular su consumo calórico. Otros estudios han evaluado el patrón alimentario de la rata a partir de la disponibilidad de alimentos con diferente contenido calórico bajo diferentes condiciones experimentales (Capaldi, Campell, Sheffer y Bradford, 1987; Young y Shuford, 1955). Sin embargo, los efectos de modificar el contenido energético de los alimentos después de un periodo de privación es un fenómeno que no ha sido suficientemente estudiado. Con base en esta evidencia se evaluó experimentalmente el efecto de modificar la cantidad de calorías disponibles en la fase posterior a la privación. Para alcanzar este objetivo se agregó una alternativa energética. Es necesario considerar que los alimentos que contienen calorías también contienen sabor. Estudios previos han investigado el efecto del sabor dulce sin calorías sobre la emisión de respuestas conductuales utilizando la sacarina (Mook, 1974; Sheffield y Thornton, 1949). Otros estudios han evaluado el efecto de la consecuencia post-ingestiva con algunos endulzantes como la glucosa o sucrosa sobre el patrón alimentario (Capaldi, Campell, Sheffer y Bradford, 1987; Rothschild, 1971). Capaldi, Owens y Palmer (1994) estudiaron los efectos de la privación 
de comida sobre las preferencias condicionadas de sacarina y sucrosa, a partir de sus propiedades de sabor y contenido energético. Concluyeron que los efectos postingestivos son determinantes para observar una preferencia específica por un endulzante.

Por otro lado, en diversos estudios en los que se utilizaron endulzantes, se ha señalado que el uso de soluciones facilita el control de los procedimientos utilizados en el estudio de los patrones alimentarios (Guttman, 1954; Lichtenstein, 1958). A partir de esta evidencia se consideró conveniente utilizar diferentes concentraciones de agua con glucosa como variable experimental para estudiar la ocurrencia de los efectos post-privación con una fuente de energía alterna.

Se diseñó un experimento con el objetivo general de evaluar experimentalmente los efectos de diferentes concentraciones de glucosa sobre el peso corporal, el consumo de alimento, el consumo de agua y el consumo de calorías durante el periodo post-privación. Un objetivo adicional fue analizar la conducta de regulación energética ante modificaciones en el número de calorías durante el periodo post-privación. Es importante señalar que estos experimentos no tienen como objetivo analizar la preferencia entre soluciones con glucosa.

El interés general se dirige a resolver las siguientes interrogantes: ¿cómo se modifican los patrones de consumo de alimento y agua en el periodo postprivación ante diferentes concentraciones de glucosa en agua?, ¿la disponibilidad de glucosa en agua afecta al peso corporal durante el periodo postprivación?, ¿es posible afectar la ocurrencia de los efectos post-privación a partir de la presencia de glucosa en el agua? y ¿qué distribución se observaría en el consumo de alimento, agua y calorías?. Se esperaba encontrar diferencias en el patrón alimentario de los animales durante el periodo postprivación respecto a los periodos previos de restricción energética, así como la presencia de grandes bebidas de agua con glucosa.

\section{MÉTODO}

\section{Sujetos}

Venticuatro ratas de la cepa Wistar, doce machos (ML1, ML2, ML3, ML4, ML5, ML6, ML7, ML8, ML9, MC10, MC11 y MC12) y doce hembras (HL1, HL2, HL3, HL4, HL5, HL6, HL7, HL8, HL9, HC10, HC11 y HC12), con 4 meses de edad al inicio del experimento e ingenuas experimentalmente.

Aparatos y Materiales

Se utilizaron veinticuatro cajas habitación individuales, con medidas de $13 \mathrm{~cm}$ de altura por $27 \mathrm{~cm}$ de ancho y $38 \mathrm{~cm}$ de largo, con una reja metálica en la 
parte superior con división para comedero y bebedero. Para el registro del consumo de alimento y peso corporal se utilizó una báscula electrónica de precisión. Croquetas de la marca comercial Nutri-cubos, especial para animales de laboratorio, fue el alimento proporcionado. Su fórmula nutricional es la siguiente: $3 \%$ de grasas, $7 \%$ de cenizas, $1 \%$ de calcio, $23 \%$ de proteína, $6 \%$ de fibra, $49 \%$ de E.L.N. (extracto libre de nitrógeno), $6 \%$ de fósforo y $12 \%$ de humedad. Como bebida se utilizó agua y durante la manipulación experimental se proporcionó una solución compuesta de glucosa diluida en agua en tres diferentes concentraciones: alta, media y baja. La concentración alta (A) fue de $45 \mathrm{~g}$ de glucosa diluidos en $200 \mathrm{ml}$ de agua, que aportó 180 calorías en total; la concentración media (M) fue de $30 \mathrm{~g}$ de glucosa en $200 \mathrm{ml}$ con 120 calorías, y la concentración baja (B) se conformó por $15 \mathrm{~g}$ de glucosa por cada $200 \mathrm{ml}$ de agua con 60 calorías. El líquido se proporcionó en bebederos graduados de $200 \mathrm{ml}$.

\section{Procedimiento}

Los sujetos fueron identificados con un número de registro, fecha de nacimiento y peso corporal al inicio del experimento y fueron colocados en cajas habitación individuales. El peso corporal, el consumo de agua y comida se registró diariamente a las 9:00 de la mañana. Para el pesaje se tomaba la caja habitación y se trasladaba a la mesa de trabajo en la que se encontraba la báscula utilizada para el registro. Se tomaba a la rata y se introducía en el recipiente de la báscula con lo que se obtenía el peso corporal. Al finalizar este procedimiento los sujetos retornaban a su caja habitación y permanecían en el bioterio.

Para calcular las calorías consumidas en el agua con glucosa y en el alimento se determinó el número de calorías que aporta el alimento por gramo a partir de la media estandarizada en nutrición animal (Subcommittee on Laboratory Animal Nutrition, Committee of Animal Nutrition, Board on Agriculture, National Council, 1995) de 3.4 calorías por gramo consumido. Esta cantidad se multiplicó por el número de gramos consumidos diariamente por el animal. El agua proporcionó 0 calorías. Cuando se adicionó glucosa al agua, se calculó el número de calorías que aportaba cada concentración de glucosa, el cual se multiplicó por el número de mililitros consumidos diariamente (Tabla 1). Se sumó la cantidad de calorías consumidas en el alimento más el número de calorías consumidas en la bebida para obtener el consumo total diario de calorías. Es necesario señalar que el protocolo fue aprobado por el comité científico de nuestra dependencia universitaria.

\section{Diseño experimental}

Se formaron tres grupos de sujetos experimentales y un grupo control. Cada uno se integró con tres hembras y tres machos. La asignación de los sujetos a cada grupo se realizó de forma aleatoria. 
El experimento se conformó de diez fases. En las fases 1 (15 días), 4, 7 y 10 (10 días c/u) se proporcionaron $50 \mathrm{~g}$ de nutricubos y $200 \mathrm{ml}$ de agua natural (libre acceso sin glucosa). En las fases 2, 5 y 8 ( 3 días c/u) se privó de alimento y los sujetos solo tuvieron disponible $200 \mathrm{ml}$ de agua (privación de alimento y de calorías). En las fases 3, 6 y 9 (5 días $\mathrm{c} / \mathrm{u}$ ), los grupos experimentales retornaron a la condición de libre acceso, pero se adicionó la glucosa al agua en las tres diferentes concentraciones (libre acceso con glucosa). El grupo Alta recibió la concentración alta de glucosa, el grupo Media recibió la concentración media y el grupo Baja la concentración baja. Los sujetos control no recibieron glucosa en la bebida durante todo el experimento. En la tabla 2 se representan las tres condiciones experimentales utilizadas.

\begin{tabular}{|c|c|c|c|c|}
\hline \multicolumn{2}{|c|}{ Condiciones experimentales } & comida & bebida & total \\
\hline \multicolumn{2}{|c|}{ Libre acceso sin glucosa } & 170 & 0 & 170 \\
\hline \multicolumn{2}{|l|}{ Privación } & 0 & 0 & 0 \\
\hline \multirow{3}{*}{$\begin{array}{l}\text { Libre acceso con } \\
\text { glucosa }\end{array}$} & Alta & 170 & 180 & 350 \\
\hline & Media & 170 & 120 & 290 \\
\hline & Baja & 170 & 60 & 230 \\
\hline
\end{tabular}

Tabla 1. Distribución de calorías disponibles. Muestra la distribución de las calorías disponibles en las tres condiciones experimentales y en cada concentración de agua con glucosa.

\begin{tabular}{|c|c|c|c|c|c|c|}
\hline \multicolumn{7}{|c|}{ Condiciones experimentales } \\
\hline Grupos & 1 & 2 & 3 & 4 & 5 & 6 \\
\hline 1-Alta & \multirow{4}{*}{$\begin{array}{c}\text { Libre } \\
\text { acceso } \\
\text { sin glucosa } \\
\text { Chow+agua }\end{array}$} & \multirow{4}{*}{ Privación } & Chow+Alta & \multirow{4}{*}{$\begin{array}{c}\text { Libre } \\
\text { acceso } \\
\text { sin glucosa } \\
\text { Chow+agua }\end{array}$} & \multirow{4}{*}{ Privación } & Chow+Alta \\
\hline 2-Media & & & Chow+Media & & & Chow+Media \\
\hline 3- Baja & & & Chow+Baja & & & Chow+Baja \\
\hline Control & & & Chow+agua & & & Chow+agua \\
\hline Días & $1-15$ & $16-18$ & $19-23$ & 24-33 & $34-36$ & $37-41$ \\
\hline
\end{tabular}

Tabla 2. Diseño experimental. Muestra el diseño experimental de las cuatro primeras fases, que se replicaron en dos ocasiones. Durante las fases 1 , 4,7 y 10 los sujetos fueron expuestos a períodos de libre acceso sin glucosa (chow+agua). En las fases 2, 5 y 8 fueron sometidos a un programa de privación de alimento durante tres días. En las fases 3, 6 y 9 se expuso a los sujetos experimentales a periodos de libre acceso con glucosa en tres concentraciones durante cinco días (chow+agua con glucosa). El grupo control se mantuvo bajo condiciones alternadas de libre acceso sin glucosa y privación durante todo el experimento. 


\section{RESULTADOS}

La Figura 1 muestra los promedios del registro diario del peso corporal de los grupos experimentales y control, la Figura 2 muestra el promedio de consumo de alimento, y la Figura 3 del consumo de agua. Las líneas continuas representan las fases de línea base y libre acceso sin glucosa $(1,4,7$ y $10)$. Las fases de privación (2, 5 y 8 ) se representan con círculos negros. Los círculos blancos representan las fases de libre acceso con glucosa (3, 6 y 9). En cada figura se muestran los datos de los grupos experimentales: Alta, Media y Baja en los paneles 1, 2 y 3 respectivamente. En la columna izquierda y derecha se representan los datos de hembras y machos de cada grupo respectivamente. El panel inferior muestra los datos del grupo control. Finalmente, la Figura 4 muestra el promedio de consumo de calorías durante cada fase del experimento.

La Figura 1 muestra el promedio del registro diario del peso corporal de cada grupo. Los sujetos experimentales y los controles presentaron una curva de crecimiento del peso corporal similar. Durante las fases de privación todos los sujetos disminuyeron su peso corporal. Cuando se adicionó la glucosa, no se observaron diferencias en la recuperación gradual del peso corporal entre los sujetos experimentales y los sujetos control, excepto en el grupo Baja, en el que los machos aumentaron su peso corporal durante las fases de libre acceso con glucosa respecto a las fases de libre acceso sin glucosa.

El consumo de alimento se presenta en la Figura 2. Todos los grupos modificaron su patrón de consumo en los periodos post-privación. Durante las fases de libre acceso sin glucosa el promedio de consumo de las hembras de los grupos experimentales se situó entre los 15 y $20 \mathrm{~g}$. Durante la privación el consumo decrementó hasta cero y durante las fases en las que se adicionó glucosa al agua se observó una tendencia a disminuir gradualmente el consumo de alimento de $15 \mathrm{a} 5 \mathrm{~g}$. Adicionalmente se observaron diferencias entre los grupos. Las hembras de los grupos Alta y Media disminuyeron un promedio de $5 \mathrm{~g}$ en su consumo de alimento respecto al promedio observado en las fases en las que no recibieron glucosa. Las hembras del grupo Baja conservaron el mismo promedio de consumo durante estas fases. Por su parte, los machos que conformaron los grupos experimentales mantuvieron un promedio de consumo de $25 \mathrm{~g}$ durante las fases de libre acceso sin glucosa (1, 4, 7 y 10); durante la privación (fases 2,5 y 8 ) el consumo de alimento cayó hasta cero y durante las fases de libre acceso con glucosa $(3,6$ y 9 ) se observaron diferencias entre los promedios de consumo. En el grupo Alta, los machos disminuyeron su consumo de alimento durante las fases de libre acceso con glucosa respecto a las fases de libre acceso sin glucosa en un promedio de $10 \mathrm{~g}$. El grupo media disminuyó su consumo en un promedio de $4 \mathrm{~g}$ durante las mismas fases. El grupo Baja mantuvo el mismo promedio de 

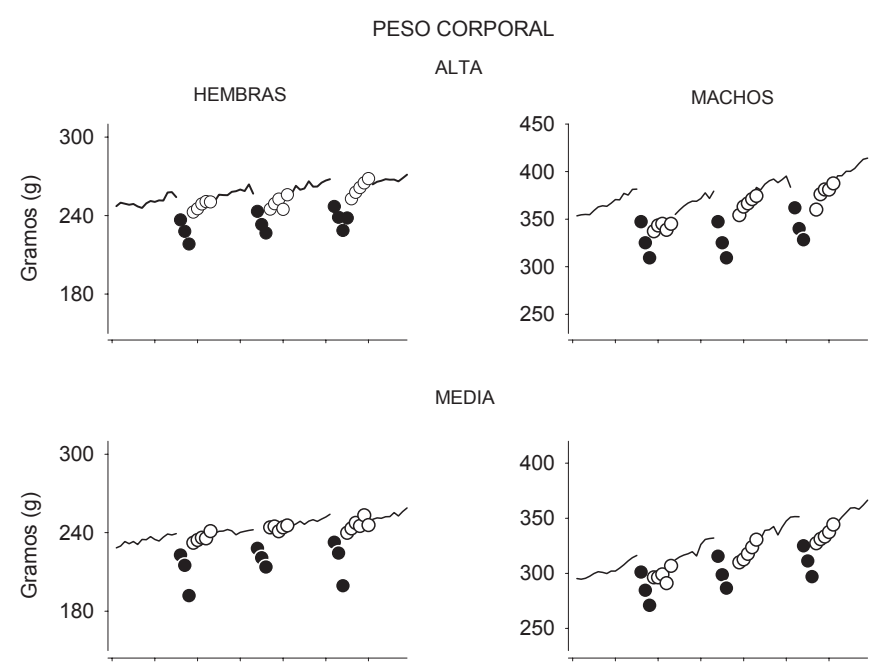

BAJA
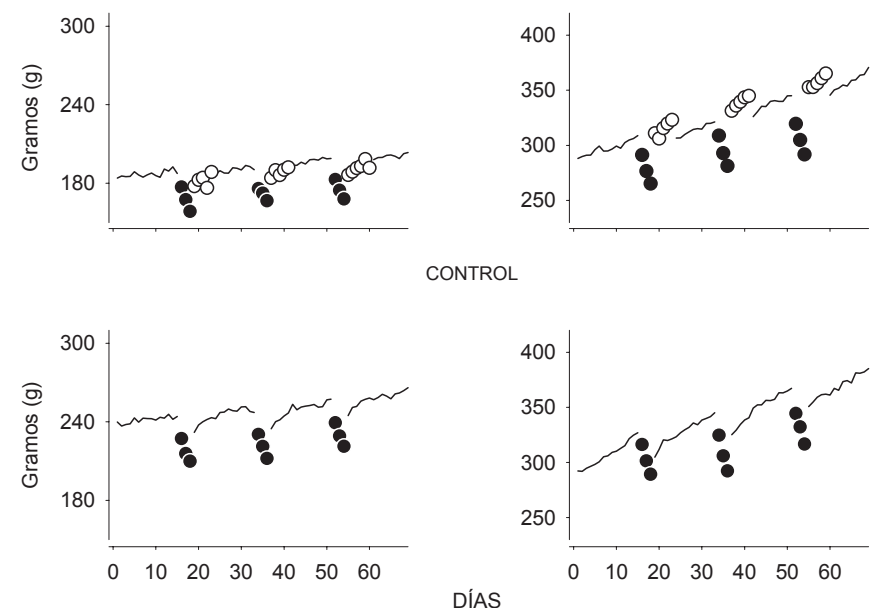

Figura 1. Promedio del peso corporal. Se presentan los datos de las hembras y los machos en las columnas izquierda y derecha respectivamente. Los grupos Alta, Media, Baja y Control se presentan en orden descendente en los paneles 1, 2, 3 y 4. La línea continua representa las fases de línea base y libre acceso sin glucosa, los círculos negros las fases de privación de alimento y los círculos blancos las fases de libre acceso con glucosa. 

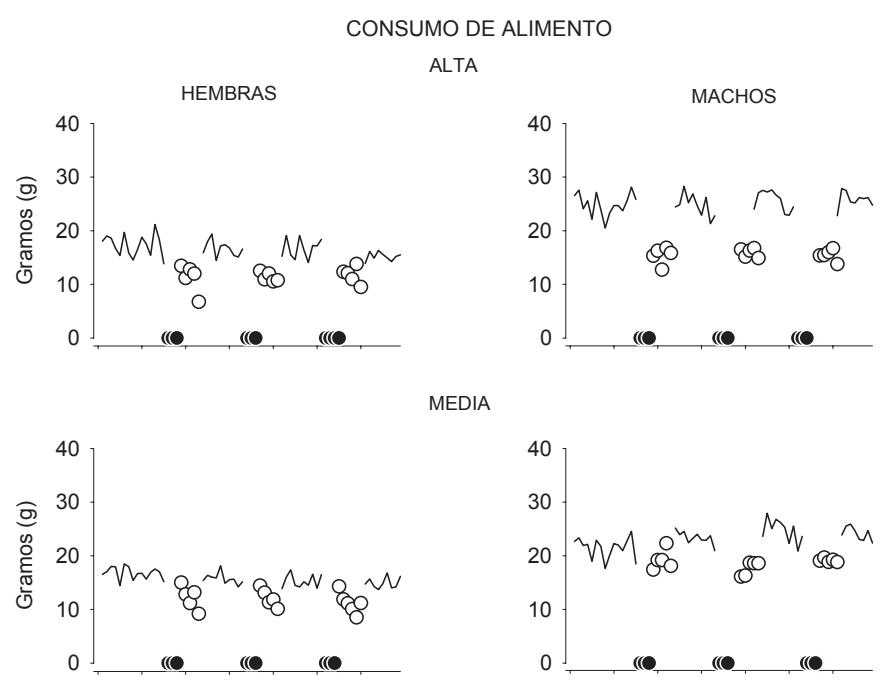

BAJA
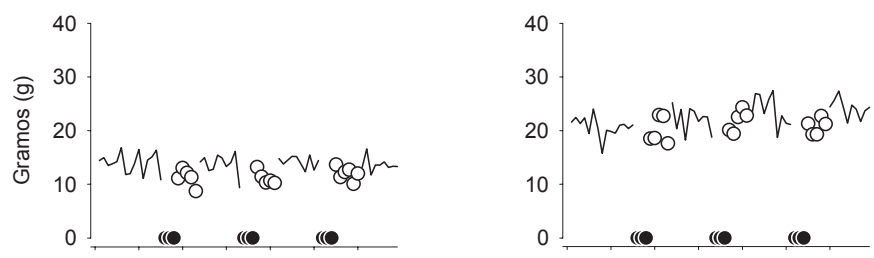

CONTROL
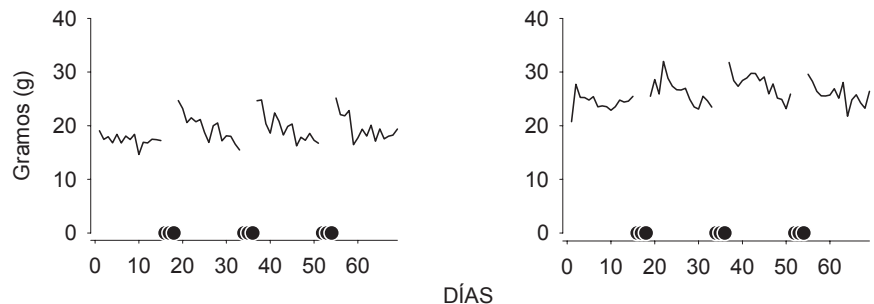

Figura 2. Promedio del consumo de alimento. Se presentan los datos de las hembras y los machos en las columnas izquierda y derecha respectivamente. Los grupos Alta, Media, Baja y Control se presentan en orden descendente en los paneles 1, 2, 3 y 4. La línea continua representa las fases de línea base y libre acceso sin glucosa, los círculos negros las fases de privación de alimento y los círculos blancos las fases de libre acceso con glucosa. 
consumo de $23 \mathrm{~g}$ durante las fases de libre acceso con y sin glucosa. Respecto a la ocurrencia de grandes comilonas en los grupos experimentales, no se presentó ninguna en los 5 días posteriores a la privación (fases de libre acceso con glucosa). Finalmente, los grupos control mostraron un promedio de consumo de $17 \mathrm{~g}$ (hembras) y $25 \mathrm{~g}$ (machos) durante la línea base, mientras que en las fases posteriores a la privación se presentaron grandes comilonas de $27-29 \mathrm{~g}$ (hembras) y $30-33 \mathrm{~g}$ (machos) en los primeros días del periodo post-privación.

La Figura 3 muestra el consumo de agua. Las hembras mostraron diferencias de consumo respecto a los machos. Durante la fase 1 , los grupos experimentales mantuvieron un promedio de consumo de $30 \mathrm{ml}$ (hembras) y $50 \mathrm{ml}$ (machos). En las fases correspondientes a la privación de alimento, el consumo de agua decrementó un promedio de $20 \mathrm{ml}$, excepto en las hembras del grupo Alta, cuyo consumo de agua fue similar al observado durante la línea base. Durante las fases en las que se adicionó glucosa al agua se presentaron diferencias entre los grupos experimentales y entre hembras y machos. Respecto a las hembras, el grupo Alta aumentó un promedio de $20 \mathrm{ml}$ su consumo de agua respecto a las fases en las que no recibieron glucosa, el grupo Media aumentó un promedio de $40 \mathrm{ml}$ y el grupo Baja un promedio de $70 \mathrm{ml}$. El aumento en el consumo de agua con glucosa de las hembras experimentales fue gradual, llegando a duplicarse, triplicarse y cuadriplicarse el promedio de consumo en los grupos Alta, Media y Baja respectivamente, en el último día de cada una de las 3 fases de libre acceso con glucosa. Por su parte, los machos experimentales también mostraron un aumento gradual en el consumo de agua con glucosa, a excepción del grupo Alta, que mantuvo el mismo promedio de consumo que el observado en las fases de libre acceso sin glucosa. El grupo Media aumentó su consumo $10 \mathrm{ml}$ y el grupo Baja $20 \mathrm{ml}$. El grupo control consumió un promedio de $30 \mathrm{ml}$ (hembras) y $50 \mathrm{ml}$ (machos) durante las fases sin glucosa y un promedio de $15 \mathrm{ml}$ (hembras) y $25 \mathrm{ml}$ (machos) durante las fases correspondientes a la privación.

Finalmente se muestra el consumo de calorías en la Figura 4. Cada barra representa el promedio de consumo de calorías durante las 7 fases del experimento en que a los sujetos se les proporcionó alimento. Las barras negras representan el consumo de calorías aportado por el alimento en cada fase del experimento y las barras blancas el consumo de calorías aportado por el agua con glucosa (fases 3,6 y 9). Los datos del grupo control se presentan mediante las barras de color gris en el panel inferior. El promedio de consumo de calorías durante las fases de libre acceso sin glucosa $(1,3,5$ y 7$)$ fue de 60 calorías en hembras y 80 en machos en los grupos experimental y control. Durante las fases de libre acceso con glucosa, el promedio de consumo de calorías aumentó a 80 y 90 en hembras y machos respectivamente. Con la adición de la glucosa se observaron diferencias en el consumo de calorías del alimento y agua 

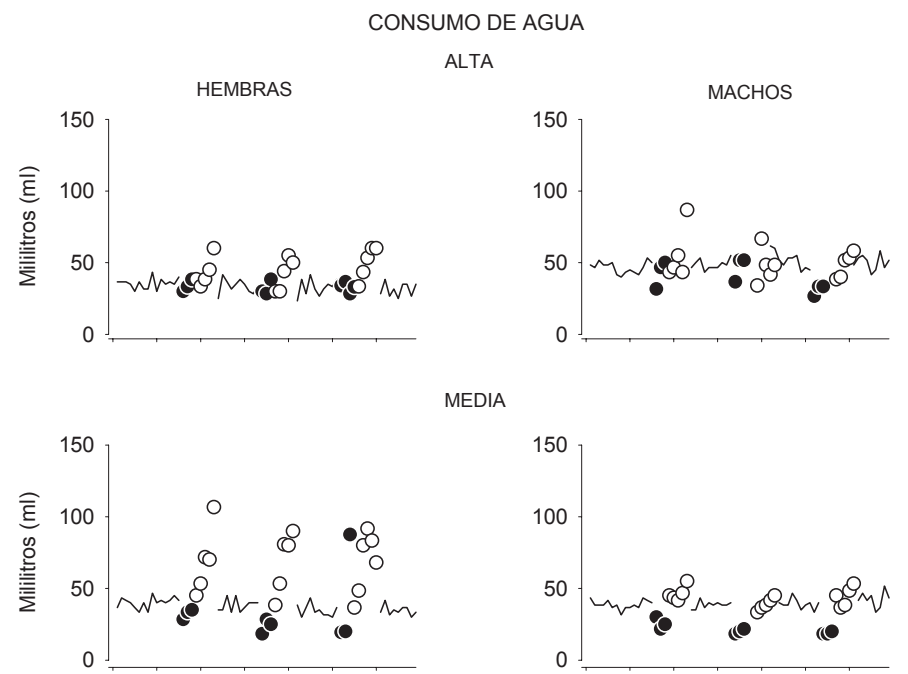

MEDIA

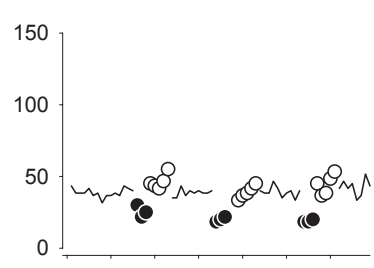

BAJA
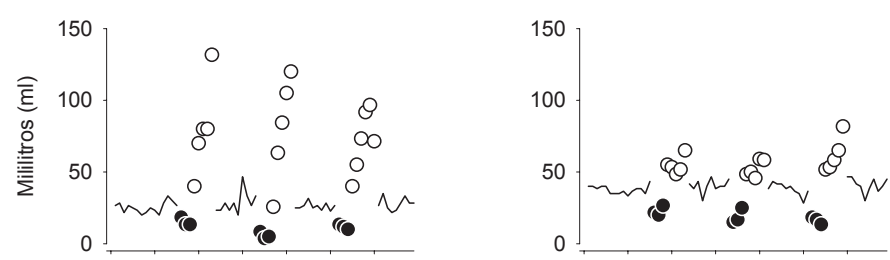

CONTROL
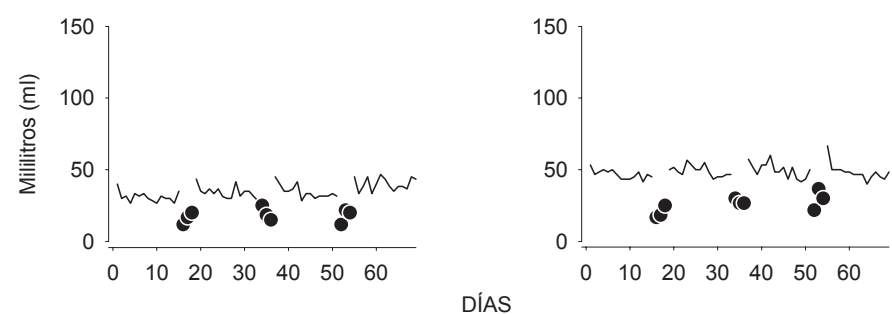

Figura 3. Promedio del consumo de agua. Se presentan los datos de las hembras y los machos en las columnas izquierda y derecha respectivamente. Los grupos Alta, Media, Baja y Control se presentan en orden descendente en los paneles 1, 2, 3 y 4. La línea continua representa las fases de línea base y libre acceso sin glucosa, los círculos negros las fases de privación de alimento y los círculos blancos las fases de libre acceso con glucosa. 

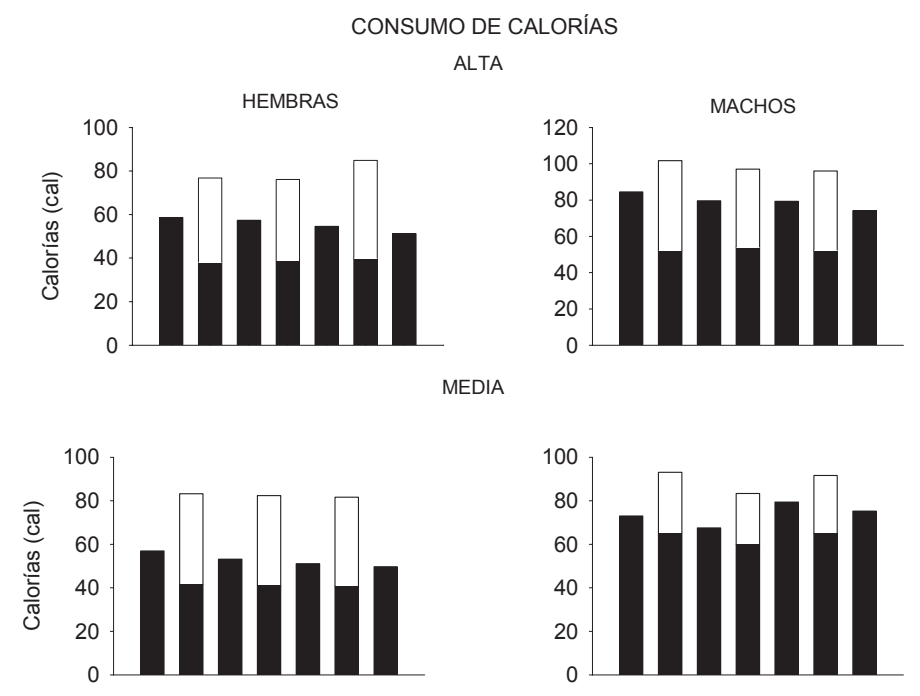

BAJA
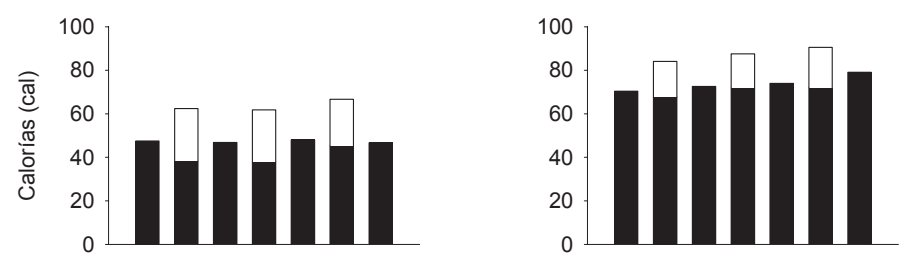

CONTROL
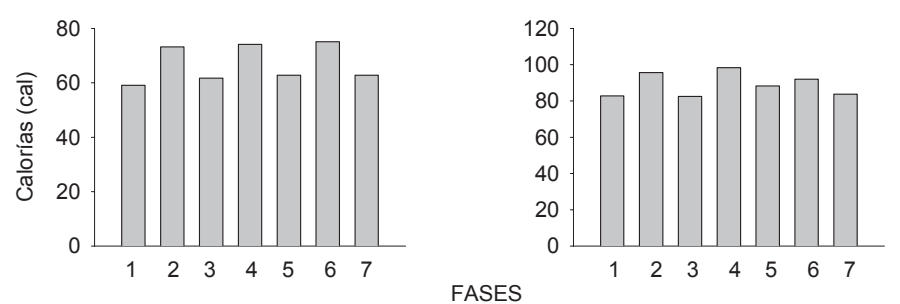

Figura 4. Promedio de consumo de calorías. Cada barra representa el promedio de consumo de calorías de las diez fases del experimento. Las barras bicolores representan el promedio de consumo durante las fases 2, 4 y 6. El color negro representa el consumo de calorías en la comida y el blanco de la bebida. Las barras grises representan el promedio de consumo de calorías del grupo control. 
entre los grupos experimentales y entre hembras y machos. Las hembras de los grupos Alta y Media consumieron un promedio de 40 calorías de alimento y 40 calorías de agua con glucosa, mientras que el grupo Baja consumió en promedio 60 calorías durante estas fases, obteniendo 40 calorías del alimento y 20 de la glucosa. Por su parte, los machos del grupo Alta consumieron un promedio de 100 calorías cuando se adicionó la glucosa (55 del alimento y 45 del agua con glucosa), el grupo Media 90 calorías (70 del alimento y 20 del agua con glucosa) y el grupo Baja 95 calorías (70 del alimento y 15 del agua con glucosa). Las hembras y machos del grupo control consumieron 60 y 80 calorías en promedio durante las fases previas a la privación y 70 y 95 calorías en las fases correspondientes al periodo post-privación respectivamente.

\section{DISCUSIÓN}

Los resultados obtenidos mostraron que el peso corporal de los sujetos experimentales: 1) disminuyó durante el periodo de privación y posteriormente fue recuperado durante las fases de libre acceso con glucosa; 2) a pesar de que el consumo de calorías fue mayor, no modificó su curva de crecimiento cuando se adicionó la glucosa al agua; y, 3) mostró el mismo patrón ascendente en su curva de crecimiento en las fases de libre acceso con glucosa que en las fases de libre acceso sin glucosa.

López-Espinoza (2001, 2004a, 2004b) y López-Espinoza y Martínez (2001a y 2001b) señalaron que una de las alteraciones conductuales presentada por los animales que son sometidos a programas de privación, es la recuperación gradual del peso corporal perdido durante el retorno a las condiciones de libre acceso. Sin embargo, los sujetos experimentales y los controles mostraron el mismo patrón de pérdida y recuperación del peso corporal. Es decir, la adición de las calorías no parece tener ningún efecto en el patrón de la curva de crecimiento del peso corporal, pues los resultados obtenidos son similares a los reportados por López-Espinoza $(2001,2004)$ que en su procedimiento experimental no ofreció otra fuente adicional de energía.

Los cambios en el peso corporal de los sujetos después de la aplicación de un programa de privación han sido reportados por diversos autores (Dimitrou, Rice y Corwin, 2000; Iwasaki, Inoue, Kiriike y Hikiji, 2000; Hagan y Moss, 1997; Lopez-Espinoza, 2001, 2004ª , 2004b; López-Espinoza y Martínez, 2001a, 2001b; Polivy y Herman, 1985). Del Prete, Balkowski y Scharrer (1994) mostraron que la restricción de comida produce aproximadamente un $25 \%$ de pérdida de peso corporal. Polivy y Herman (1985) y López-Espinoza (2001, 2004a, 2004b) señalaron que las modificaciones en el peso corporal de los sujetos experimentales involucran cambios drásticos en el consumo de alimento y agua. 
El diseño experimental utilizado en este experimento permitió observar cambios en el patrón de consumo de alimento, agua y agua con glucosa y diferencias de consumo entre los grupos experimentales. Se observó que: 1) el consumo de alimento disminuyó gradualmente con la adición de la glucosa, 2) el consumo de agua con glucosa aumentó gradualmente, 3) el promedio de consumo de alimento y agua durante las fases sin glucosa se mantuvo estable, y, 4) los sujetos control presentaron grandes comilonas y los sujetos experimentales grandes bebidas.

López-Espinoza (2001, 2004a, 2004b) señaló que en el periodo posterior a la privación de alimento se observa un incremento en el consumo de alimento identificado como gran comilona. Los sujetos experimentales no mostraron grandes comilonas, lo que sugiere que la disponibilidad de otra fuente de energía más palatable permite a la rata distribuir su consumo de calorías de tal forma que no se observen grandes consumos de alimento. Del Prete, Balkowski y Scharrer (1994) señalaron que las ratas incrementan su ingesta de comida voluntariamente durante la primera semana después de la privación, respecto a sus controles. Mencionaron que se incrementa el tamaño de la comida, pero no el número de comidas. Nuestros resultados mostraron que también se modifica el número de calorías consumidas.

Los resultados sugieren que en los grupos experimentales, la ausencia de grandes comilonas se sustituyó por la presencia de grandes bebidas a partir de la distribución en el consumo de calorías. Los sujetos experimentales duplicaron, triplicaron y cuadriplicaron su consumo de agua con glucosa gradualmente, alcanzando consumos de hasta $150 \mathrm{ml}$ en promedio (hembras del grupo Baja). López-Espinoza (2001, 2004a) reportó grandes bebidas de 70 y $80 \mathrm{ml}$ en el periodo post-privación. La diferencia entre los consumos de agua parece involucrar dos características: el sabor y las calorías utilizadas en la manipulación experimental. Durante el retorno a las condiciones de libre acceso las ratas mantuvieron disponible un sabor agradable y además una consecuencia post-ingestiva, en los experimentos de López-Espinoza (2001, 2004a) estas propiedades no estuvieron presentes.

Nisbett (1972) sugirió que un organismo privado tiende a consumir más comida "buena" proporcionalmente en comparación a uno no privado. Reportó que cuando las ratas son privadas de comida y se le da acceso a agua y agua con sacarina, muestra una marcada preferencia por la solución endulzada respecto a los sujetos control (no privados de comida). Por su parte, Valenstein (1967) mostró que los animales no privados a quienes se les ofrecen dos soluciones con glucosa: una altamente dulce y una menos dulce, prefieren la menos dulce. Lo que explicaría por qué el grupo Baja mostró un consumo mayor que el del grupo Alta y Media. Sin embargo, esta conducta solo fue observada en las hembras, que presentaron mayores consumos de agua con glucosa con respecto a los machos. Estas diferencias en el 
consumo se han reportado anteriormente en pruebas de preferencia (Mook, 1974). Adicionalmente, Duffy y Bartoshuk (1996) y Raynor y Epstein (2001) señalaron que las mujeres son más responsivas ante los sabores dulces y amargos con respecto a los hombres y argumentaron que en esta diferencia se involucran los niveles hormonales.

Con base en estas evidencias, los datos obtenidos sugieren que los animales privados prefieren el alimento con mejor sabor, más dulce o palatable. Adicionalmente, el patrón alimentario de los sujetos experimentales parece relacionarse con el número de horas de privación para que muestren este tipo de conducta. Probablemente los animales privados muestren más habilidades discriminativas en su alimentación. Del Prete, Balkowski y Scharrer (1994) señalaron que la restricción de comida produce hambre específica por carbohidratos. Estudiaron la selección de nutrientes en ratas después de 5 días de restricción de alimento ( $5 \mathrm{~g}$ de alimento disponibles diariamente), observaron que el consumo de carbohidratos se incrementó mientras que el consumo de grasa se mantuvo constante. Estas evidencias explicarían por qué los sujetos experimentales mostraron grandes bebidas respecto al consumo del grupo control, así como la disminución en el consumo del alimento sólido.

Los resultados obtenidos en el presente estudio advierten la necesidad de alcanzar un mejor conocimiento sobre el control experimental de la conducta reguladora de la alimentación. Es necesario profundizar en la investigación de las propiedades subyacentes de los alimentos como responsables de ciertas respuestas alimentarias. De igual forma, el estudio de procedimientos como las privaciones sucesivas de alimento o agua representa un instrumento eficaz para determinar los efectos acumulativos de la restricción, como los efectos post-privación.

\section{REFERENCIAS}

Anliker, J., y Mayer, J. (1957). The regulation of food intake: some experiments relating behavioral, metabolic and morphologic aspects. American Journal of Clinical Nutrition, 5, 148-153.

Baker, R. A. (1955). The effects of repeated deprivation experience on feeding behavior.

Capaldi, E. D. (1996). Conditioned food preferences. En E. D Capaldi (ed). Why we eat what we eat. USA: American Psychological Association, 53-80.

Capaldi, E. D., Campell, D. H., Sheffer, J. D., y Bradford, J. P. (1987). Conditioned flavor preferences based on delayed caloric consequences. Journal of Experimental Psychology: Animal Behavior Processes, 13, 150-155.

Capaldi, E. D., Owens, J., y Palmer, K. A. (1994). Effects of food deprivation on learning and expresión of flavor preferentes condicioned by saccharin or sucrose. Animal Learning \& Behavior, 22, 173-180. 
Collier, G., Hirsh, E., y Kanareck, R. (1983). La operante vista de Nuevo. En W. K. Honig, y J. E. R. Staddon (eds.). Manual de conducta operante. México: Trillas.

Corwin, R. L., Wojnicki, F. H. E., Fischer, J. O., Dimitrou, S. G., Rice, H. B., y Young, M. A. (1998). Limited access to a dietary fat option affects ingestive behavior but not body composition in male rats. Physiology and Behavior, 65, 545-553.

Del Prete, E., Balkowski, G., y Scharrer, E. 1994). Meal pattern of rats during hyperfhagia induced by long-term food restriction is affected by diet composition British Journal of Nutrition, 23, 79-86.

Dimitrou, S. G., Rice, H. B., y Corwin, J. (2000). Effects of limited access to a fat option on food intake and body composition in female rats. International Journal of Eating Disorders. John Wiley \& son's Inc.

Duffy, V. B., y Bartoshuk, L. M. (1996). Sensory factors in feeding. En E. D Capaldi (ed). Why we eat what we eat. USA: American Psychological Association, 143171.

Guttman, N. (1954). Equal-reinforcement values for sucrose and glucose solutions compared with equal sweetness values. Journal of Comparative and Physiological Psychology, 47, 358-361.

Hagan, M., M. y Moss, D. E. (1997). Persistence of binge-eating patterns after a history of restriction with intermittent bouts of refeeding on palatable food in rats: Implications for bulimia nervosa. International Journal of Eating Disorders, 22, 411-420.

Iwasaki, S., Inoue, K., Kiriike, N. y Hikiji, K. (2000). Effect of maternal separation on feeding behavior of rats in later life. Physiology and Behavior, 70, 551-556.

Keesey, R. E. (1986). A set-point theory of obesity En K. D. Brownell y J. P. Foreyt (Eds.), Handbook of eating disorders. (pp. 63-87) New York: Basic Books., Publishers.

Lane, M. A., Ingram, D. K., y Roth, G. S (1999). Nutritional modulation on aging in nonhuman primates. The Journal of Nutrition, Health y Aging, 3, 69-78.

Lawrence, D. H., y Masson, W. A. (1955). Food intake in the rat as a function of deprivation intervals and feeding rhythms. Journal of Comparative and Physiological Psychology, 48, 267-271.

Lichtenstein, P. E. (1958). The relative sweetness of sugars: sucrose and dextrose. Journal of Comparative and Physiological Psychology, 38, 278-286.

López-Espinoza, A. (2001). Efectos de la privación de agua y comida sobre el peso corporal y el consumo de alimento y agua en ratas albinas (Rattus norvegicus). Tesis de maestría inédita, Universidad de Guadalajara, Jalisco, México.

López-Espinoza, A. (2004a). El fenómeno alimentario: ¿un problema biológico o psicológico?. Cuadernos de Nutrición, 27, 173-177.

López-Espinoza, A. (2004b). Análisis experimental de los efectos post-privación. Una propuesta para el control de la gran comilona en ratas albinas (Rattus norvegicus). Tesis doctoral inédita, Universidad de Guadalajara, Jalisco, México.

López-Espinoza, A. y Martínez, H. (2001a). Efectos de dos programas de privación alimentaria sobre el peso corporal de ratas Wistar. Revista Mexicana de Análisis de la Conducta, 27, 35-46.

López-Espinoza, A. y Martínez, H. (2001b). Efectos de dos programas de privación parcial sobre el peso corporal y el consumo total de agua y comida en ratas. Acta 
Comportamentalia, 9, 5-17.

López-Espinoza, A. y Martínez, H. (2004). Cambios en el patrón alimentario como efecto de la privación de agua o alimento en ratas en crecimiento. International Journal of psychology and psychological therapy, 4, 93-104.

Mayer, J. (1955). Regulation of energy intake and body weight. The glucostatic theory and the lipostatic hypothesis. Annals of the New York Academy of sciences, 63, 15-43.

Mook, D. G. (1974). Saccharin preference in the rat: some unpalatable findings. Psychological Review, 81, 475-490.

Nisbett, R. (1972). Hunger, obesity, and the ventromedial hypothalamus. Psychological Review, 79, 433-453.

Polivy, J. y Herman, P. (1985). Dieting and binging: A causal analysis. American Psychologist, 40, $193-201$.

Raynor, H. A. y Epstein, L. H. (2001). Dietary variety, energy regulation, and obesity. Psychological Bulletin, 127, 325-341.

Rothschild, G. H. (1971). Patterns of concentration preference for glucose and sodium chloride in rats. The psychological record, 21, 487-496.

Sheffield, F. D., y Thornton, R. (1950). Reward value of a non-nutritive sweet taste. Journal of Comparative and Physiological Psychology, 43, 471-481.

Siegel, P. S. y Stuckey H. L. (1947). The diurnal course of water and food intake in the normal mature rat. Journal of Comparative and Physiological Psychology, 40, 365-370.

Staddon, J. E. R., y Zanutto, B. S. (1998). In praise of parsimony. En D. L. Wynne y J. E. R. Staddon (eds.). Models of action. USA: Lawrence Erlbaum Associates Publishers.

Strubbe, J. H., y Woods, S. C. (2004). The timming of meals. Psychological Review, $111,148-121$.

Subcommittee on Laboratory Animal Nutrition, Committee of Animal Nutrition, Board on Agriculture, National Research Council. (1995). Nutrient Requirements of Laboratory En línea : National Academies Press.

Valenstein, E. (1967). Selection to a nutritive and nonnutritive solutions under different conditions of need. Journal of Comparative and Physiological Psychology, 63, 429-433.

Weingarten, H. P. (1990). Learning, homeostasis and control of feeding behavior. En E. D. Capaldi, y T. L. Powley (eds.). Taste, experience \& feeding: development and learning. USA: American Psychological Association.

Young, P. T., y Shuford, E. H. (1955). Quantitative control of motivation through sucrose solutions of different concentrations. Journal of Comparative and Physiological Psychology, 48, 114-118. 